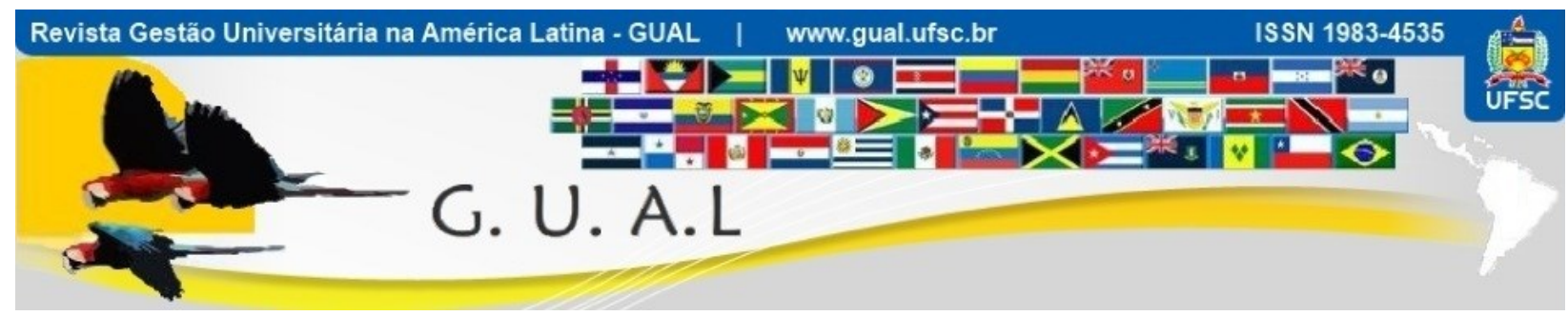

DOI: http://dx.doi.org/10.5007/1983-4535.2016v9n1p278

\title{
MODELO PARA ANÁLISE DE CAPACIDADE DE PROJETOS DE PESQUISA DE UMA INSTITUIÇÃO DE ENSINO SUPERIOR
}

\author{
MODEL FOR CAPACITY ANALYSIS IN RESEARCH PROJECTS AT HIGHER \\ EDUCATION INSTITUTION
}

\author{
Marcelo Benedetti, Graduado \\ Universidade de Caxias do Sul - UCS \\ benedettimarcelo@yahoo.com.br \\ Gabriel Vidor, Doutor \\ Universidade de Caxias do Sul - UCS \\ gvidor@ucs.br
}

Recebido em 10/setembro/2014

Aprovado em 13/novembro/2015

Sistema de Avaliação: Double Blind Review

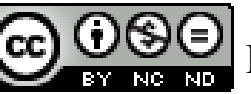

Esta obra está sob uma Licença Creative Commons Atribuição-Uso. 


\title{
RESUMO
}

As instituições de ensino superior no Brasil estão em forte crescimento devido à demanda de profissionais pela indústria e à exigência de novas tecnologias. Para explorar esse crescimento e se tornar referência em seu segmento, as instituições vêm se aprofundando em metodologias aplicadas nas indústrias para se tornarem mais competitivas. Desse modo, o presente artigo tem por objetivo desenvolver um modelo para analisar a capacidade de execução em projeto de pesquisa de uma instituição de ensino superior. Esse modelo aplica os conceitos de capacidade, método simplex e modelagem de cenários para geração de cenários, resultando em um índice de maximização da taxa da margem de contribuição total da capacidade. Esse resultado é um indicativo de viabilidade do projeto em análise. A validação desse estudo foi realizada pela análise de um grupo de projetos do laboratório de pesquisa ambientais de uma instituição de ensino superior. No final do estudo pode-se constatar a eficiência do modelo para a tomada de decisão, demonstrando que é possível gerar cenários que foquem em aspectos importantes para o determinado contexto.

Palavras-chaves: Capacidade. Método Simplex. Modelagem de cenários.

\begin{abstract}
Higher education institutions in Brazil are growing strongly due to demand by industry professionals and the demand for new technologies. To exploit this growth and become a benchmark in its segment, the institutions have been deepening in methodologies applied in industries to become more competitive. Thus, this article aims to develop a model to analyze the execution capabilities in research project of a higher education institution. This model applies concepts capability, simplex method and modeling scenarios for generating scenarios, resulting in a maximization of the ratio of the rate margin total capacity contribution. This result is indicative of viability of the project under review. The validation of this study was conducted by analyzing a group of environmental research laboratory projects of a higher education institution. At the end of the study can be seen the model of efficiency for decision making, demonstrating that it is possible to generate scenarios that focus on important aspects for the given context.
\end{abstract}

Keywords: Capacity. Simplex Method. Scenario Modeling. 


\section{INTRODUÇÃO}

As instituições de ensino superior (IES) brasileiras estão enfrentando dificuldades em sua gestão, decorrente da utilização de metodologias que não conseguem reagir às constantes flutuações econômicas e tecnológicas do mundo atual (CUNHA, 2011). As instituições estão recebendo um número crescente de procura de vagas pela sociedade (INEP, 2011), o que consequentemente aumenta as suas receitas, mas contraditoriamente não estão aptas a competir devido sua complexidade organizacional (VIDOR; FOGLIATTO; CATEN, 2013). De fato, observa-se que essa estrutura complexa, decorre da visão estagnada presente nas universidades, onde técnicas de gestão, aplicadas em empresas brasileiras de sucesso, não transitam facilmente no universo do ensino superior. A introdução de técnicas de gestão pode trazer benefícios socioeconômicos, transformando a organização, tornando-a competitiva em relação a seus concorrentes e dando um retorno em um patamar internacional para a sociedade que está em constante procura de seu bem (CUNHA, 2011).

Segundo Andrade (2006 apud MAINARDES; MIRANDA; CORREIA 2001), as universidades baseadas na teoria de recursos e capacidade, tem maior probabilidade de êxito em relação à gestão com enfoque externo. Portanto, a capacidade do sistema define os limites competitivos da empresa, determinando a taxa de resposta da empresa ao mercado, sua estrutura de custo, a composição de sua força de trabalho, seu nível de tecnologia, suas exigências de gestão e de apoio ao quadro funcional.

Um exemplo de aplicação dessas técnicas é a utilização da análise de capacidade para modelagem de cenários. Gaither e Frazier (2002) mostram em seu estudo que a capacidade de produção pode ser empregada em produtos e serviços, visto que objetiva obter o maior nível de produção de uma empresa, dentro da estrutura programada para o trabalho realizado. Capacidade excessiva pode ser tão agonizante quanto a sua insuficiência, além disso, pode haver uma oscilação entre ambas ao longo do tempo. As instabilidades, políticas e econômicas, são dadas devido à dinamicidade global, onde é necessário lidar com uma demanda variável e incerta. Devido a essas incertezas, pode-se paralelamente utilizar a técnica da modelagem de cenários, pois muitas vezes os valores reais são apenas estimados. $\mathrm{Na}$ utilização dos valores estimados, a análise pode determinar o quanto os valores ótimos das variáveis de decisão serão afetados (KRAJEWSKI; RITZMAN, MALHOTRA, 2009) e, portanto, fortalecer o processo de tomada de decisão nas IES. 
Desse modo, este artigo abordará o desenvolvimento de um modelo de capacidade em projeto de pesquisa para as IES, para ajudá-las nas mudanças de cenário que vêm enfrentado, decorrente de uma gestão defasada que não absorve as exigências de uma sociedade em constante evolução. A instituição, com a implantação desse modelo, torna-se mais competitiva em relação a seus concorrentes, pois sua capacidade produtiva irá atender a demanda de forma eficaz, e irá reduzir os custos do departamento, independentemente da situação econômica em que está inserida.

\section{REFERENCIAL TEÓRICO}

O referencial teórico está organizado por meio de duas partes. A primeira engloba uma descrição de casos de estudo em pesquisas de gestão universitária. A segunda trata da ferramenta de otimização linear usada no caso de análise desse estudo.

\subsection{ESTUDOS E PESQUISAS NA GESTÃO UNIVERSITÁRIA}

O estudo realizado por Venturini et al. (2010), na Universidade Federal de Santa Maria (UFSM), traz o processo de avaliação institucional da UFSM. Para o desenvolvimento, os dados da pesquisa foram coletados utilizando o método escalar desenvolvido por Likert. Solicitou-se, a cada docente entrevistado, a escolha da alternativa, entre as apresentadas, que melhor expressasse sua percepção em relação à avaliação da universidade e sobre os fatores que nela interferem (pesquisa caracterizada do tipo descritiva). Além dos dados primários, obtidos por meio de questionário respondido pelos docentes, também foram utilizados dados secundários obtidos por meio da análise dos seguintes documentos: Projeto de Avaliações da UFSM, Projeto Político-Pedagógico e relatórios elaborados pela Comissão Central de Avaliação. Os resultados da pesquisa permitem afirmar que, na percepção da maioria dos docentes, o processo de avaliação institucional em andamento na UFSM é considerado como bom ou regular. Entretanto, $79,9 \%$ e $95,7 \%$ dos fatores estudados facilitam ou dificultam o processo de avaliação, destacando-se com mais de $70 \%$ os seguintes: consciência da importância da avaliação, definição clara dos critérios e indicadores a serem utilizados na avaliação, definição e divulgação das políticas da instituição e a existência de comissão interna de avaliação.

Práticas de Gestão de Conhecimento na Gestão Universitária foi o tema do estudo realizado por Pereira et al. (2011). Esse estudo foi realizado na Universidade do Estado do 
Rio Grande do Norte (UERN) com o objetivo de identificar quais práticas de Gestão de Conhecimento são utilizadas na Gestão Universitária. Para o seu desenvolvimento, utilizou-se uma abordagem quantitativa inserida numa pesquisa descritiva-exploratória, na medida que os dados foram observados, registrados, analisados e interpretados. Ao analisar as práticas e ferramentas da gestão do conhecimento, foi verificado que a instituição utiliza em seu setor de Gestão de Pessoas as seguintes estratégias: um portal corporativo; um programa de treinamento e capacitação; incentivo ao trabalho em equipe e a estratégia de benchmarking. Essas práticas permitem que as informações sejam disseminadas no interior e exterior da organização promovendo também uma disseminação de conhecimentos ao serem absorvidos pelos seus usuários. Essa dispersão de informações/conhecimento possibilita melhor formação dos profissionais na medida em que abrange a discussão sobre determinados assuntos.

O estudo realizado por Mainardes, Miranda e Correia (2011), foi desenvolvido em três organizações de três cidades distintas (Joinville, Blumenau e Brusque). Em Joinville, foi pesquisada a Faculdade Cenecista de Joinville (FCJ); em Blumenau, foi pesquisado o Instituto Blumenauense de Ensino Superior (IBES); em Brusque, foi pesquisada a Faculdade do Vale do Itajaí Mirim (FAVIM). Este estudo objetivou identificar como é realizada a gestão estratégica em instituições particulares de ensino superior de pequeno e médio porte em Santa Catarina, comparando-a com a gestão estratégica em micro, pequenas e médias empresas (MPME), no intuito de se encontrar similaridades e divergências. Para o desenvolvimento, foram realizados três estudos de caso, com a aplicação de pesquisas exploratórias qualitativas transversais com os principais gestores das IES, por meio de entrevista pessoal apoiada em um roteiro que continha vinte e oito assuntos relevantes, os quais visaram tipificar a gestão estratégica das IES estudadas. Essa entrevista, que pode ser caracterizada como despadronizada ou não estruturada. Com os resultados, identificou-se características e comportamentos semelhantes entre si na gestão estratégica das instituições pesquisadas, bem como similares aqueles próprios da gestão estratégica de MPME. Embora as IES sejam consideradas organizações complexas, muitas características próprias na gestão estratégica em MPME são perfeitamente aplicáveis às instituições privadas de pequeno porte.

O estudo realizado por Dos Santos e Bronnemann (2013) teve como objetivo analisar os desafios de gestão a partir da percepção de diretores de centro de uma instituição de ensino pública do sul do Brasil. Os temas abordados pela pesquisa foram: conhecimentos, avaliações de outras pessoas, tempo disponível, comprometimento dos subordinados, gestão de 
divergências, burocracia e centralização, e cuidados éticos. A partir do estudo, utilizaram uma abordagem qualitativa de caráter descritivo-interpretativa, por meio de um estudo de caso único seguindo as orientações de Stake, para que o critério de maior importância consista em maximizar o que se pode aprender por meio dele. Os resultados da pesquisa afirmam que os principais desafios enfrentados pelos diretores de centro estão relacionados à ausência de conhecimento prévio sobre a função e sobre aspectos de gestão; ao tempo disponível para o cargo em relação ao número de atividades que necessitam ser realizadas; e à gestão de divergências relacionais de subordinados, administrativas e pessoais.

O estudo realizado por Vidor, Fogliatto e Caten (2013), na Universidade Federal do Rio Grande do Sul (UFRGS), teve como objetivo propor uma sistemática para avaliar cursos de pós-graduação em IES. A sistemática busca identificar, por meio de um questionário, critérios inibidores da qualidade e promotores da qualidade. Critérios significativos nos dois blocos são identificados por meio de uma Análise Fatorial. Em um segundo passo, esses critérios são contemplados através de ações que atendem as demandas identificadas. O trabalho realizado é do tipo quantitativo, por meio do método de estudo de caso, no qual conduziu uma pesquisa descritiva do tipo survey. Os pesquisadores concluíram que a ferramenta proposta permite identificar pontos de insatisfação e focar melhorias, especificamente para os clientes (docentes e discentes) de programas de pós-graduação. Além disso, proporciona um canal direto para comunicação entre a gestão, seus docentes e discentes e, também, institui um ciclo de melhorias para diferentes metas que possam ser elaboradas por uma instituição.

Os estudos realizados em diversas universidades demonstraram a necessidade de constantes e crescentes pesquisas relacionadas ao desenvolvimento sócio educacional nas IES, para que as mesmas consigam ofertar e atender os serviços de forma adequada diante ao mercado competitivo. Os pesquisadores citados obtiveram êxito na aplicação e obtenção dos resultados, demonstrando que as metodologias aplicadas nas empresas atuais podem ser empregadas nas instituições de ensino com sucesso.

\subsection{MODELO DE OTIMIZAÇÃO}

O método simplex foi desenvolvido após a segunda guerra mundial, por George B. Dantzig. Entre 1941 e 1945, Dantzig trabalhou no Pentágono como especialista em planejamento e programação de atividades militares, em seguida, na mesma organização 
trabalhou como conselheiro em matemática da Força Aérea. O desenvolvimento do método simplex se deu a partir da necessidade de um método que mecanizasse o processo de planejamento, mas com algumas características: ser dinâmico, ter flexibilidade e ser computacionalmente tratado (COLIN, 2011).

O método simplex é uma importante ferramenta destinada a resolver problemas de programação linear (PL). Esse método consiste em buscar, caso existe (m), uma (ou mais) solução (ões), partindo-se de uma solução básica factível e gerando uma sequência de soluções factíveis. Quando essa sequência é completada, a solução ótima é obtida (BARBOSA; ZANARDINI, 2010).

Lisboa (2002, p. 15 apud BARBOSA; ZANARDINI, 2010) afirma que esse método “caminha pelos vértices da região viável até encontrar uma solução que não possua soluções vizinhas melhores que ela. Esta é a solução ótima”. O autor alerta que há duas situações em que não é possível chegar à solução ótima, esses casos ocorrem quando: (i) não há uma solução que se deve ou possa executar por apresentar restrições de incompatibilidade; (ii) não encontrar um máximo ou um mínimo. Nesse caso, ocorre quando uma das variáveis pode se estender ao infinito, consequentemente terá um valor sem limites para a função objetivo.

A forma padrão de problemas de programação linear garante que as entradas do algoritmo simplex tenham a mesma estrutura. Para o algoritmo de maximização, a notação matricial pode ser definida como:

$$
\begin{aligned}
& \operatorname{maximizar} \mathrm{z}=\mathrm{c}^{\mathrm{T}} x \\
& \text { sujeito a: } A x=\mathrm{b} \\
& x \geq 0 .
\end{aligned}
$$

Para a notação matricial, variáveis artificiais de folga e excesso necessárias já foram adicionadas, então, todas as restrições estão na forma $=$. Portanto, $A$ é uma matriz $m \times n$ (isto é, $m$ linhas e $n$ colunas), $b$ é um vetor de $m, c$ e $x$ são $n$ vetores, assim as variáveis $n$ (representado como $x$ ) inclui a folga / excesso / variáveis artificiais. Suponha que se sabe que as variáveis $n$ são básicas no quadro ideal, uma vez que existem restrições $m$, haverá variáveis básicas $m$ e $(n-m)$ variáveis não básicas. Colocando todas variáveis básicas juntas bem como, todas as variáveis não básicas divide-se o vetor $x$ em $\left[\frac{x_{B}}{x_{N}}\right]$, onde $x_{\mathrm{B}}$ denota as variáveis 
básicas $m$, e $x_{\mathrm{N}}$ as variáveis não básicas. Posteriormente, também o grupo de colunas de $A$ correspondente as variáveis $x_{\mathrm{B}}$ na matriz $m \times m$ de $B$, e as colunas de base não restantes para a $m \times(n \times m)$ matriz $N$. O vetor de custo é dividido de forma semelhante como $c^{T}=\left[\begin{array}{ll}c_{B}^{T} & c_{N}^{T}\end{array}\right]$ Assim, o quadro de partida para o método simplex pode ser escrito da seguinte forma (ARENALES et al., 2007):

\begin{tabular}{|c|c|c|c|}
\hline$Z$ & $\boldsymbol{x}_{B}$ & $\boldsymbol{x}_{N}$ & rhs \\
\hline 1 & $-c_{B}^{T}$ & $-c_{N}^{T}$ & 0 \\
\hline 0 & $\mathbf{B}$ & $\mathrm{N}$ & $\mathbf{b}$ \\
\hline
\end{tabular}

Quadro 1 Quadro de partida para o Método Simplex.

Fonte: ARENALES et al., (2007).

\section{MATERIAIS E MÉTODOS}

Para responder à questão de pesquisa tratada nesse estudo, o método de trabalho está organizado por meio de cinco fases. Essas fases são apresentadas no Quadro 2.

\begin{tabular}{|c|c|c|c|c|}
\hline \multicolumn{5}{|c|}{$\begin{array}{l}\text { Desenvolver um modelo de maximização da taxa da margem de contribuição total da capacidade em } \\
\text { projeto de pesquisa de uma instituição de ensino superior. }\end{array}$} \\
\hline \begin{tabular}{l}
\multicolumn{1}{c}{ Fase 1 } \\
- Identificar as \\
variáveis que \\
afetam o projeto \\
- Identificar o \\
gasto com cada \\
variável \\
- Resultado: \\
informações para \\
otimização
\end{tabular} & $\begin{array}{l}\quad \text { Fase } 2 \\
\text { - Fomular o } \\
\text { modelo } \\
\text { - Calcular a } \\
\text { capacidade atual } \\
\text { - Resultado: } \\
\text { maximização da } \\
\text { taxa da margem } \\
\text { de contribuição } \\
\text { total da } \\
\text { capacidade }\end{array}$ & \begin{tabular}{l}
\multicolumn{1}{c}{ Fase 3} \\
- Modelagem de \\
cenários. \\
- Simular cenários. \\
- Resultado: \\
cenários atual, \\
pessimista, otimista \\
e realista.
\end{tabular} & \begin{tabular}{l}
\multicolumn{1}{c}{ Fase 4} \\
- Definir processo \\
para auxiliar a \\
tomada de decisão \\
do diretor de centro \\
- Resultado: \\
processo de tomada \\
de decisão definido
\end{tabular} & \begin{tabular}{l}
\multicolumn{1}{c}{ Fase 5} \\
- Acompanhar o \\
andamento dos custos \\
de capacidade do \\
projeto \\
- Resultado: Relatório \\
mensal de \\
acompanhamento dos \\
custos de capacidade
\end{tabular} \\
\hline
\end{tabular}

Quadro 2 Etapas do trabalho.

Fonte: Elaborado pelo autor (2015).

$\mathrm{Na}$ primeira fase são identificadas as variáveis do projeto de pesquisa a serem analisadas. Essas variáveis são conhecidas como variáveis restritivas de capacidade. O cenário desse artigo, a capacidade está associada às questões de disponibilidade das pessoas envolvidas no projeto e disponibilidade dos insumos do laboratório usados no projeto. Para responder a essas questões de capacidade, é necessário verificar a alocação atual das pessoas envolvidas e quanto tempo podem dedicar ao projeto. Já no caso dos insumos, identificam-se quais serão utilizados no projeto e, em seguida, verifica-se a sua disponibilidade. Com essas 
informações calcula-se o gasto com cada uma das variáveis identificadas. Sendo assim, o principal resultado dessa fase é a geração das informações de entrada para o modelo de otimização.

$\mathrm{Na}$ segunda fase é possível calcular o total de disponibilidade que maximiza o uso da capacidade na operacionalização do projeto. Sendo assim, essa fase tem por objetivo aplicar um modelo de otimização para determinar a taxa da margem de contribuição total da capacidade. Para esse cálculo é utilizada a ferramenta Solver do Excel 2013.

$\mathrm{Na}$ fase seguinte, com a capacidade, receitas e custos identificados é possível verificar como incrementos de informações podem modificar a situação atual. Esses incrementos de informações podem ser o acréscimo de pessoas e tempo dos insumos do laboratório, ou a redução de pessoas e tempo dos insumos do laboratório.

Com as informações necessárias é possível gerar o cenário atual, pessimista, otimista e realista. Para gerar o cenário atual não será necessário simular as informações, já para o cenário pessimista terá um acréscimo de cinco por cento na receita e vinte por cento nos custos. No caso do cenário otimista, terá um acréscimo de trinta por cento na receita e dez por cento nos custos e, por fim, para o cenário realista terá um valor meta de acordo com os índices anuais de dissídio da classe e reajuste de matrícula. Esses percentuais estão apresentados no Quadro 3.

\begin{tabular}{|l|c|c|}
\hline \multicolumn{1}{|c|}{ Cenário } & Receita (\%) & Custo (\%) \\
\hline Atual & 0 & 0 \\
\hline Pessimista & 5 & 20 \\
\hline Otimista & 30 & 10 \\
\hline Realista & Meta & Meta \\
\hline
\end{tabular}

Quadro 3 Percentuais para geração dos cenários.

Fonte: Elaborado pelo autor (2015).

A elaboração dos cenários é realizada por meio de modelagem de cenários. Nessa modelagem de cenários, as variáveis restritivas do problema são modificadas a partir das informações definidas pela direção do centro (como relatado anteriormente, os percentuais podem ser modificados para simular o que é considerado interessante pela direção de centro). Os resultados dessa fase mostram cenários que auxiliam a direção de centro na tomada de decisão sobre a viabilidade dos projetos.

$\mathrm{Na}$ quarta fase, o objetivo é definir um processo para auxiliar o diretor do centro na aprovação ou reprovação do projeto de pesquisa. Essa decisão está baseada nos cenários 
gerados para o projeto em análise e comparada com os demais projetos de pesquisa existentes no centro onde a decisão deve ser tomada. Salienta-se que a alteração de atividades impacta sobre o funcionamento do centro, as demais atividades são de cunho institucional e dependem de modificações no âmbito da gestão da universidade. Dessa forma, as atividades que não estão no escopo das atividades do gestor dos projetos de pesquisa, não serão tratadas nesse trabalho.

$\mathrm{Na}$ fase final, o processo de acompanhamento e controle de projeto é realizado pelo diretor do centro. Essa é uma fase nova e não existe formalmente no processo atual. Nessa fase um acompanhamento da taxa da margem de contribuição total da capacidade é realizado mensalmente pelo diretor do centro, o que permite refinar o processo de tomada de decisão no acompanhamento do projeto de pesquisa.

O processo para tomada de decisão do diretor do centro é apresentado na Figura 1.

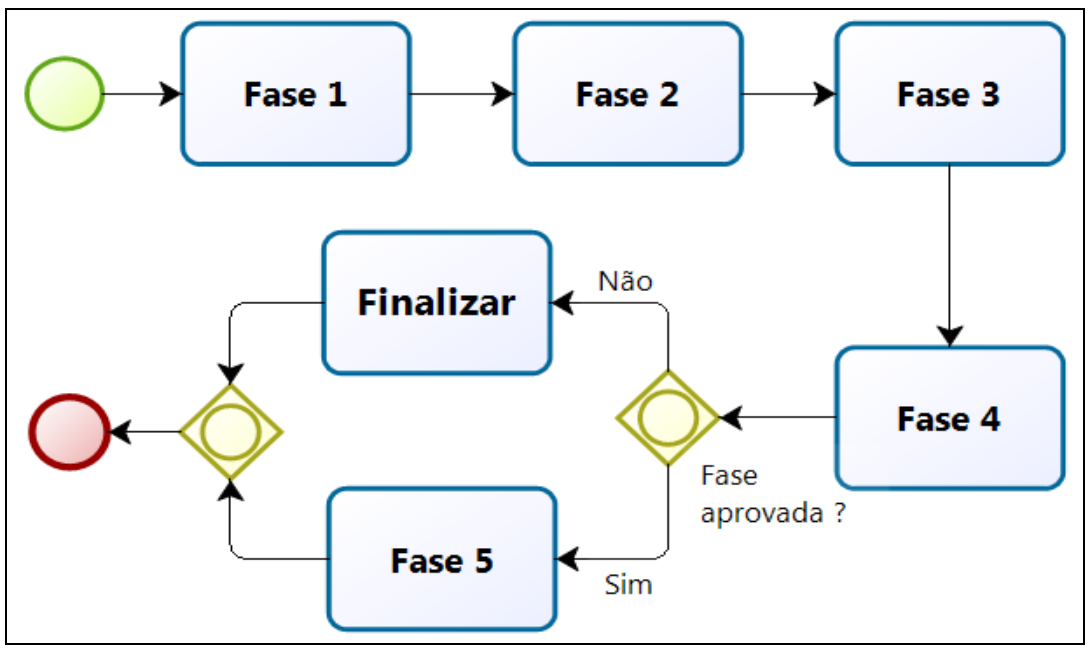

Figura 1 Processo para tomada de decisão.

Fonte: Elaborado pelo autor (2015).

Esse processo cria uma estrutura de análise que proverá direção ao diretor do centro, indicando qual o melhor caminho a seguir, com base nas variáveis restritivas de tempo e de insumos. É importante salientar que se os cenários apresentados na Fase 4 não atenderem os objetivos propostos, finaliza-se a proposta de projeto.

\section{RESULTADOS}

\subsection{DESCRIÇÃO DO CASO}

O desenvolvimento do projeto de pesquisa, na Instituição estudada, passa por cinco etapas em quatro setores até a sua aprovação ou reprovação, onde, respectivamente, as 
atividades são finalizadas e o projeto arquivado. A necessidade do projeto inicia por meio do proponente (professor), dentro de suas áreas de conhecimento/especialidade que elaboram propostas para execução de Projetos de Pesquisas. A proposta será avaliada na unidade de pesquisa estudada, em caso de reprovação é enviada ao proponente, que realizará as revisões necessárias para, em seguida, solicitar nova avaliação ao colegiado do centro. No caso de aprovação a proposta é encaminhada pelo gestor da pesquisa para sua coordenadoria, onde irá verificá-la e se caso não estiver de acordo, solicita ao proponente complementar a sua proposta. E se a proposta estiver de acordo será enviada ao Comitê Assessor de Pesquisa (e quando necessário aos consultores ad-hod externos do CNPq).

$\mathrm{Na}$ verificação da proposta, podem ocorrer três situações: (i) solicitar reformulação; (ii) solicitar reformulação para o próximo semestre; (iii) aprovar. Ao solicitar a reformulação, a proposta é devolvida à Coordenadoria de Pesquisa, diretamente ao professor/coordenador, que deverá efetuar as alterações solicitadas e remetê-la novamente à Coordenadoria de Pesquisa e a mesma avalia aprovando ou reprovando a proposta. Caso de reprovação, será enviado o pedido ao proponente para cancelar as atividades.

Ao pedir reformulação para o próximo semestre, a proposta será devolvida ao professor que poderá submeter a proposta novamente no semestre seguinte, no entanto ela percorrerá todas as etapas do processo. Quando a proposta é aprovada, será remetida a informação que o projeto poderá ser executado. Os projetos, cuja execução prática ou experimentação envolva seres humanos serão encaminhados ao Comitê de Ética em Pesquisa da Instituição, após aprovação de mérito pelas instâncias anteriores será liberado para o desenvolvimento.

Esse cenário apresenta uma visão burocrática e filosófica que deixa de considerar as estratégias de produção empregadas no cotidiano das organizações. Essas estratégias definem de forma lógica se o projeto em questão será rentável, possibilitando uma análise de viabilidade econômica. Portanto, a Instituição terá um retorno financeiro esperado, quando aplicar metodologias que forneçam resultados para a tomada de decisão sobre a rentabilidade de um projeto. Por exemplo, simulações com grupos de variáveis, visando obter o menor custo operacional de um projeto de pesquisa. Dessa forma, dentre os benefícios principais destaca-se o retorno financeiro assegurado do seu investimento transformando-se em um centro sólido, autônomo (em relação aos custos) e tecnologicamente estruturado; a 
possibilidade de prestar serviços externos de forma segura sem eventuais surpresas com relação à capacidade destinada para determinada atividade.

\subsection{CRIAÇÃO DO MODELO}

Essa seção apresenta inicialmente a descrição das variáveis identificadas como relevantes no problema de otimização dos recursos da unidade de pesquisa estudada. Essas variáveis são agrupadas da seguinte forma: (i) pessoas, esse grupo consiste de duas variáveis. A primeira a quantidade de horas $(\mathrm{PH})$ e a segunda o valor hora (PV). A quantidade de horas está relacionada à alocação necessária de recursos da Instituição para atender um determinado projeto. Sabendo isso, é possível programar e direcionar a demanda de projetos, utilizando os funcionários da melhor forma possível. $\mathrm{O}$ valor hora é o custo hora total dos envolvidos no projeto, podendo oscilar decorrente do número e da categoria dos profissionais envolvidos; (ii) equipamentos, composto também de duas variáveis. A quantidades de horas $(\mathrm{EH})$, que está relacionada ao tempo total de utilização dos equipamentos para o desenvolvimento do projeto. O valor hora $(\mathrm{EV})$ que corresponde ao custo total da utilização dos equipamentos; (iii) matéria-prima envolve outras duas variáveis. A quantidade de matéria-prima (MPQ) e valor da matéria-prima (MPV). A quantidade de matéria-prima corresponde a todos os materiais necessários para executar o projeto e atingir o objetivo da pesquisa. $\mathrm{O}$ valor é o custo total de todos os materiais utilizados em todos os estágios do desenvolvimento; (iv) laboratório contempla as variáveis de quantidade de horas $(\mathrm{LH})$ e o valor hora (LV). A quantidade de horas do laboratório está relacionada à quantidade de tempo de ocupação de um determinado espaço. O valor é o custo destinado a cada área ocupada pelo projeto na instituição; (v) fornecedores possui apenas a variável de valor (FV). O valor é o custo total de serviços externos prestados ao projeto de pesquisa. Há projetos que demandam essas atividades, porque a instituição não possui recurso ou tempo hábil para executá-las.

Conhecidas essas informações, foi possível modelar o sistema de soluções para esse estudo. O primeiro passo foi identificar nessas variáveis a sua contribuição em termos de resultado para uma instituição. Nesse sentido todas as variáveis são tratadas como indicadores de receita e custo. Além disso, o foco desse estudo é criar um modelo para análise de capacidade de execução dos projetos de pesquisa, o que implica na modelagem de uma função objetivo que determine a viabilidade de operação de um projeto de pesquisa. 
Nesse sentido a função objetivo proposta deve maximizar os recursos disponíveis, indicando se os recursos geram capacidade ou não. Os $x_{i}$ recursos são analisados de acordo com a Equação 1. Essa equação indica que um resultado positivo viabiliza a operação do projeto de pesquisa em um recurso, enquanto que o resultado negativo mostra a inviabilidade de operacionalização do projeto de pesquisa.

$$
M A X z=\sum_{i=1}^{n} a x_{i}
$$

onde $x_{i}$ indica as $n$ variáveis de decisão para a função objetivo.

No caso como existe uma operacionalização entre receitas e custos, o que se busca é maximizar a margem de contribuição de cada projeto de pesquisa para uma determinada área. Se a margem global dos $n$ recursos dos projetos for positiva, o laboratório ou centro de pesquisa justifica-se. Além do coeficiente $x_{i}$, a função objetivo é composta por um fator de carga $a$ para recurso. O cálculo do fator de carga vem apresentado na Equação 2.

$$
a=\frac{\sum_{i=1}^{n} y_{i}}{D}
$$

onde $D$ representa a disponibilidade do recurso no período de análise.

Nesse estudo a disponibilidade representa a capacidade disponível para a realização das tarefas. Além disso, yi indica os valores de receita e custo para os i períodos de análise. Conhecida as variáveis de decisão e a função objetivo, torna-se necessário modelar as restrições do problema. As restrições são definidas em função da agregação de valor gerada. Quando o impacto é positivo, a restrição de um valor meta deve ser maximizado e quando o impacto é negativo o valor meta deve ser minimizado. Isso implica na modelagem condicional das restrições, como vem apresentada na Equação 3.

$x_{i}\left\{\frac{\leq \alpha \text { se, } \sum_{i=1}^{n} y_{i} \geq 0}{\geq \alpha \text { se, caso contrário }}\right.$

onde $\alpha$ representa o valor meta para o recurso $x_{i}$

Observa-se pela equação que as restrições são do tipo $\leq$ para receita e $\geq$ para as variáveis de custo. De fato, cada variável é analisada individualmente sobre uma meta estipulada, não sendo possível restringir composições de receita e custo. 
Para terceira etapa do método, uma modelagem do cenário deve ser aplicada sobre as resultantes da fase anterior. A Equação 4 traz o detalhamento, onde se observa que o valor meta de cada variável está acrescido de um valor demandado. Esse valor pode ser um objetivo da organização ou mesmo um fator de correção para gerar um cenário passado.

$x_{i}\left\{\frac{\leq \alpha+\Delta s e, \sum_{i=1}^{n} y_{i} \geq 0}{\geq \alpha+\Delta \text { se, caso contrário }}\right.$

Na sequência, a metodologia desenvolvida é aplicada em um caso de análise para mensurar a sua aderência a projetos de pesquisa, avaliando aspectos positivos e negativos da aplicação da pesquisa operacional à tomada de decisão na gestão universitária.

\subsection{ESTUDO DE CASO}

O estudo de caso foi realizado em uma unidade de pesquisa da área ambiental na Instituição estudada, com o objetivo de identificar a taxa da margem de contribuição total da capacidade utilizada. Para isso, foi criado o cenário atual, cenário pessimista, cenário otimista e o cenário realista para identificar a viabilidade do funcionamento do laboratório. Para o desenvolvimento do estudo, disponibilizou-se as informações necessárias (Relatório Comparativo do Resultado do Exercício - Orçamento vs Realizado e o Relatório Evolução Analítica Mensal Orçamentária).

O modelo foi desenvolvido a partir da identificação e redução das principais variáveis do sistema existente e seus valores convertidos em taxas, permitindo a resolução do problema de forma linear. Essa conversão permite uma padronização dos dados facilitando a compreensão das informações pelo gestor da pesquisa que pode tratar cada uma das restrições do problema como metas de otimização.

Para calcular a taxa de cada variável, primeiramente encontra-se a disponibilidade de horas do laboratório por ano, como vem apresentado na Equação 5. Essa equação refere-se a uma das variáveis da Equação 2.

$D I=h \times m=200 \times 12=2400$ horas $/$ ano

onde DI corresponde a capacidade disponível para desenvolver trabalhos no laboratório, $h$ corresponde a horas trabalhadas no mês e $m$ a quantidade de meses usados.

$\mathrm{Na}$ sequência, procedeu-se a identificação e o cálculo da taxa das variáveis, utilizando os valores dos relatórios disponíveis. Para cada grupo de rubricas do relatório, foi 
possível estimar as taxas de valores de receita e custo associados a cada variável de decisão do problema. A primeira a ser calculada foi a taxa de receita, como apresentado na Equação 6. Essa equação refere-se a aplicação da Equação 2.

$T R=\frac{\sum \operatorname{Re} \text { ceitas }}{D I}=\frac{193.513,00}{2400}=R \$ 80,63$ horas $/$ ano

onde $T R$ é a taxa de receita.

Posteriormente, as Equações 7, 8, 9, 10, 11 e 12 foram usadas para calcular as taxas de custo do laboratório.

$T M A=\frac{\text { Custos Materiais Auxiliares }}{\text { DI }}=\frac{42.450,00}{2400}=R \$ 17,68$ horas $/$ ano

onde TMA taxa com materiais auxiliares.

$T P=\frac{\text { Custo Pessoal }}{D I}=\frac{319.753,00}{2400}=R \$ 133,23$ horas $/$ ano

onde $T P$ é taxa com pessoas.

$T S T=\frac{\text { Serviços Terceirizado }}{D I}=\frac{66,00}{2400}=R \$ 0,0275$ horas $/$ ano

onde TST é a taxa com serviços terceirizados.

$T G G=\frac{\text { Gastos Gerais }}{D I}=\frac{35 \cdot 271,00}{2400}=R \$ 14,696$ horas $/$ ano

onde $T G G$ é a taxa com gastos gerais. Por gastos gerais, contemplou-se todas variáveis que tradicionalmente compõe o DRE, exceto a rubrica de conservação de equipamentos. Essa diferenciação faz-se necessária, dado o impacto gerado dessa variável na resolução desse problema.

$T E=\frac{\text { Conservação Equipamento }}{D I}=\frac{45 \cdot 165,00}{2400}=R \$ 18,818$ horas $/$ ano

onde $T E$ representa a taxa de conservação com equipamentos.

$T O D=\frac{\text { Outras Despesas }}{D I}=\frac{25.670,00}{2400}=R \$ 10,695$ horas $/$ ano

onde $T O D$ refere-se a outras despesas. Nessa taxa estão contemplados os valores de despesas com bolsas - auxílios e estágios, depreciações, despesas financeiras e despesas não operacionais.

Conhecidas as taxas, é possível iniciar a resolução do problema. O segundo passo foi modelar a função objetivo do problema seguindo a Equação 1. Dessa forma, obteve-se a Equação 13.

$M A X T M C C=\sum x_{i}$

onde $T M C C$ é a taxa de capacidade, expressa em função da margem de contribuição, $x_{i}$ corresponde a cada elemento de taxa calculado anteriormente.

Observa-se que a função objetivo é definida pela maximização da taxa da margem de contribuição da capacidade, dessa forma valores positivos indicam que o laboratório tem 
condições de operação e valores negativos indicam que o laboratório não deve permanecer aberto ou necessita de adequações para viabilizar o seu funcionamento.

$\mathrm{O}$ agrupamento de todas as informações gerou o modelo que vem apresentado na Figura 2. As variáveis de decisão são compostas por sete variáveis descritas na forma de X1 a X7. Na linha da solução, o solver do Excel registra o valor ótimo das variáveis de decisão. Para calcular o valor ótimo, os coeficientes para cada uma das restrições forma uma matriz técnica entre X1 a X7 e a $1^{\mathrm{a}}$ Restrição a $7^{\mathrm{a}}$ Restrição (com uma diagonal igual a um). Já na linha da função $Z$, são os coeficientes das variáveis de decisão (esses coeficientes são as taxas das receitas e dos custos) e, na mesma linha, na coluna da fórmula do Z, retornará o seu resultado. $\mathrm{Na}$ mesma coluna tem-se a fórmula do $1^{\circ}$ membro, serve para calcular o valor do primeiro membro para cada restrição técnica. Na coluna ao lado, são inseridos sinais orientativos para os valores de segundo membro (RHS) de cada restrição e, na coluna RHS, está os seus respectivos valores (que serão as taxas das receitas e dos custos). A coluna ao lado (os valores são expressos em reais/ano para cada restrição) e, na linha lucro, o resultado será a diferença entre a receita $\left(1^{\mathrm{a}}\right.$ restrição) e a soma dos custos (da restrição $2^{\mathrm{a}}$ a $\left.7^{\mathrm{a}}\right)$.

\begin{tabular}{|c|c|c|c|c|c|c|c|c|c|c|c|}
\hline Variáveis de Decisão & $\mathrm{X} 1$ & $\mathrm{x} 2$ & $\mathrm{x} 3$ & $\mathrm{X} 4$ & $\mathrm{X} 5$ & $\mathrm{x} 6$ & $\mathrm{X} 7$ & & \multirow{4}{*}{\multicolumn{2}{|c|}{ RHS }} & \\
\hline \multirow[t]{3}{*}{ Solução } & 80,630 & 17,680 & 133,230 & 0,028 & 14,696 & 18,818 & 10,695 & \multirow{3}{*}{ Formula do $1^{\circ}$ membro } & & & \\
\hline & & & & & & & & & & & \\
\hline & $\mathrm{X} 1$ & $\mathrm{x} 2$ & $\mathrm{X} 3$ & $\mathrm{X} 4$ & $\mathrm{X} 5$ & $\mathrm{x} 6$ & $\mathrm{x} 7$ & & & & \\
\hline $1^{a}$ Restrição & 1 & & & & & & & 80,630 & $<=$ & 80,630 & RS $193.512,00$ \\
\hline $2^{a}$ Restrição & & 1 & & & & & & 17,680 & $>=$ & 17,680 & RS $42.432,00$ \\
\hline $3^{a}$ Restrição & & & 1 & & & & & 133,230 & & 133,230 & RS $319.752,00$ \\
\hline $4^{a}$ Restrição & & & & 1 & & & & 0,0275 & $>=$ & 0,0275 & RS $\quad 66,00$ \\
\hline $5^{a}$ Restrição & & & & & 1 & & & 14,696 & $>=$ & 14,696 & RS $35.270,40$ \\
\hline $6^{\mathrm{a}}$ Restrição & & & & & & 1 & & 18,818 & $>=$ & 18,818 & RS $45.163,20$ \\
\hline \multirow[t]{3}{*}{$7^{\mathrm{a}}$ Restrição } & & & & & & & 1 & 10,695 & $>=$ & 10,695 & RS $25.668,00$ \\
\hline & & & & & & & & & & Lucro & -RS $274.839,60$ \\
\hline & & & & & & & & Formula do $\mathrm{Z}$ & & & \\
\hline $\mathrm{Z}=$ & 80,630 & 17,680 & 133,230 & 0,0275 & 14,696 & 18,818 & 10,695 & $-12246,09172$ & & & \\
\hline
\end{tabular}

Figura 2 Simulação do cenário atual.

Fonte: Elaborado pelo autor (2015).

Para simular os demais cenários propostos nesse estudo, foram utilizados a mesma metodologia de construção, mas com os seus respectivos valores já citados. O Quadro 4 representa as variáveis e os cenários simulados. O Quadro 4 mostra as variáveis e as 
flutuações apresentadas em cada uma em função dos cenários propostos. Salienta-se que os cenários apresentados seguem as recomendações apresentadas na Seção 3 desse artigo.

\begin{tabular}{|l|l|l|l|l|}
\hline VARIÁVEIS & $\begin{array}{l}\text { CENÁRIO } \\
\text { ATUAL }\end{array}$ & $\begin{array}{l}\text { CENÁRIO } \\
\text { PESSIMISTA }\end{array}$ & $\begin{array}{l}\text { CENÁRIO } \\
\text { OTIMISTA }\end{array}$ & $\begin{array}{l}\text { CENÁRIO } \\
\text { REALISTA }\end{array}$ \\
\hline$x_{1}=$ TR & 80,63 & 84,662 & 104,819 & 232,339 \\
\hline$x_{2}=$ TMA & 17,68 & 21,216 & 19,448 & 18 \\
\hline$x_{3}=$ TP & 133,23 & 159,876 & 146,553 & 133 \\
\hline$x_{4}=$ TST & 0,0275 & 0,033 & 0,03 & 1 \\
\hline$x_{5}=$ TGG & 14,696 & 17,635 & 16,166 & 15 \\
\hline$x_{6}=$ TE & 18,818 & 22,582 & 20,70 & 19 \\
\hline$x_{7}=$ TOD & 10,695 & 12,834 & 11,765 & 11 \\
\hline
\end{tabular}

Quadro 4 Valores das variáveis para os cenários simulados.

Fonte: Elaborado pelo autor (2015).

\subsection{IMPLICAÇÕES GERENCIAIS}

Para testar e simular os cenários, foram utilizadas informações de um grupo de projetos do Laboratório de Análise e Pesquisas Ambientais (LAPAM), todavia outros grupos de projetos de pesquisa podem ser utilizados. Essa característica demonstra que o método tem flexibilidade para inserir e retornar as informações esperadas pelo diretor de centro. Mesmo que o retorno de informação seja em termos qualitativos, pode-se aferir sobre a viabilidade de implementação do projeto de pesquisa. Percebe-se que se o resultado da otimização for positivo, o projeto em questão terá ganhos da taxa da margem de contribuição total da capacidade, caso contrário, se for negativo, indica perda sinalizando que é inviável desenvolver o projeto. Outro aspecto a destacar é que o resultado da maximização mostra que o resultado em termos de lucro difere do resultado em termos de ganhos, pois o importante da análise é considerar o efeito da margem de contribuição.

A utilização e a operação das informações no modelo permitem um entendimento pleno do negócio, decorrente da geração de diversos cenários. A aplicação do modelo tem por base as informações de histórico de projetos desenvolvidos, o que facilita a aplicação do projeto. Dessa forma, a tomada de decisão torna-se rápida e acurada, podendo aprovar ou reprovar consistentemente o projeto proposto. Ou seja, com a aplicação de cenários, o diretor de centro terá informações relevantes para aprovar o projeto e passará por todas as etapas já mencionadas (na seção 4.1) ou poderá finalizá-lo de imediato sem a necessidade de encaminhar para as etapas seguintes. No caso do projeto ser finalizado passará por duas etapas, reduzindo o esforço de todos os envolvidos. Além disso, outra alteração será a eliminação da atividade de reformulação para o próximo semestre da terceira etapa; o projeto 
não ficará no aguardo, pois o diretor terá as informações necessárias e relevantes para tomar a decisão de aprovar ou reprovar o projeto. Assim, o processo se torna mais eficiente e menos burocrático, devido a autonomia que o diretor terá sobre as decisões e que consequentemente irá reduzir as atividades de outros setores que estão envolvidos nessa análise. Portanto, o ganho não está apenas no setor que analisa o projeto, mas na organização, onde se economiza a força de trabalho.

Finalmente, a ferramenta permite o controle periódico (semanal, mensal, etc.) dos projetos em andamento. Esse controle é mediado pela taxa da margem de contribuição total da capacidade pelo diretor de centro, por meio da aplicação do método criado. Sendo assim, a geração de cenários possibilita também que o diretor de centro compare a situação do projeto em questão com a situação econômica da instituição. Caso o projeto esteja demandando um investimento elevado e sem retorno aparente ou a instituição esteja passando por dificuldades, poderá cancelá-lo imediatamente desde que cumpra com a sua contrapartida. Essa análise também pode ser realizada no âmbito nacional e internacional, visando os ganhos que o projeto poderá trazer para a instituição. Em alguns casos mesmo sabendo que a previsão do projeto até o seu término não trará lucro, o diretor pode mantê-lo ativo porque trará retornos a longo prazo, ou seja, pode ser uma decisão estratégica. Possibilita também a identificação de problemas relacionados aos gastos já realizados e permite prevê-los até o término do projeto. Dessa forma, o diretor do centro poderá tratar esses problemas, transformando o projeto rentável até a sua entrega. As soluções para esses problemas poderão estar voltadas ao manejo de profissionais para determinadas atividades para encontrar a melhor alocação e à utilização de materiais, equipamentos e máquinas em geral, sendo possível tratar e reverter situações do presente e, ao mesmo tempo, direcioná-las para um futuro próximo. Complementarmente uma análise gráfica de indicadores pode ser aplicada, a fim de refinar o processo para tomada de decisão. Além disso, devido à avaliação ser ininterrupta, projetos de pesquisa podem ser findados a qualquer momento.

\section{CONSIDERAÇÕES FINAIS}

Este artigo teve por objetivo desenvolver um modelo para analisar a capacidade de execução em projeto de pesquisa de uma instituição de ensino superior. Por meio do estudo foi criado um modelo que permite a maximização da taxa da margem de contribuição total da capacidade em projeto de pesquisa. Esse modelo consiste das etapas de identificação das 
variáveis restritivas do ambiente (financeiro e operacional) e geração de cenários para a tomada de decisão. Para gerar os cenários otimista, pessimista e realista foi aplicada a modelagem de cenários. A validação desse modelo foi possível por meio de um caso de estudo na Instituição que confirmou a sua viabilidade.

Com relação aos objetivos propostos inicialmente no artigo, obteve-se algumas informações importantes. Em um primeiro momento foi possível identificar os elementos restritivos de capacidade no contexto de projetos de pesquisa no ambiente de estudo. Os valores dessas restrições foram convertidos em taxas, permitindo a resolução na forma linear. Dessa forma, a padronização dos dados facilita a análise e o controle dos projetos pelo diretor.

Na sequência a utilização do modelo, com auxílio da ferramenta Solver do Excel permitiu modelar os cenários atual, pessimista, otimista e realista, quanto ao funcionamento do modelo. Salienta-se que a resposta do modelo é em termos qualitativo, o que facilita a tomada de decisão pelo gestor. Dessa forma, se for positiva indicará viabilidade do projeto; caso contrário, se for negativa, indicará inviabilidade. Cabe ao diretor decidir se manterá o projeto em funcionamento, ou se aprovará a execução do mesmo.

Enfim, o artigo apresenta uma nova sistemática para auxiliar a tomada de decisão do diretor de centro. Os projetos de pesquisa da Instituição não tinham acompanhamento e nem análise através de um modelo matemático de simulação, sendo a decisão onerada por um processo burocrático. O modelo permite ao diretor identificar a viabilidade do projeto sem passar por todas as etapas do processo, reduzindo as atividades de outros setores que estão envolvidos nessa análise, tendo um ganho não apenas local (no centro), mas organizacional (devido à economia da força de trabalho). Além disso, é eliminada a fase de reformulação para o próximo semestre da terceira etapa, pois o diretor terá informações para decidir aprovação ou reprovação do projeto no instante da análise.

\section{REFERÊNCIAS}

ARENALES, Marcos Nereu; ARMENTANO, Vinícius Amaral; MORABITO, Reinaldo; YANASSE, Horacio Hideki. Pesquisa operacional. Rio de Janeiro: Elsevier, 2007.

BARBOSA, Marcos Antonio; ZANARDINI, Ricardo Alexandre Deckmann. Iniciação à pesquisa operacional no ambiente de gestão. Curitiba: Ibpex, 2010.

COLIN, Emerson Carlos. Pesquisa operacional: 170 aplicações em estratégia, finanças, logística, produção, marketing e vendas. Rio de Janeiro: LTC, 2011. 
CUNHA, Marcos Ribeiro. Gestão Estratégica de IES: Modelos e funções do planejamento estratégico em universidades publicas e privadas de Palmas - Tocantis. Lisboa: ulusofona, 2011. Dissertação (Mestrado em Educação), Instituto de Educação, Universidade Lusófona de Humanidades e Tecnologias, 2011.

GAITHER, Norman; FRAZIER, Greg. Administração da produção e operações. 8. ed. São Paulo: Pioneira Thomson Learning, 2002.

KRAJEWSKI, Lee; RITZMAN, Larry; MALHOTRA, Manoj. Administração de produção e operações. 8. ed. São Paulo: Pearson Prentice Hall, 2009.

MAINARDES, Emerson Wagner; MIRANDA, Cristina Schmitt; CORREIA, Carlos Henrique. A Gestão Estratégica de Instituição de Ensino Superior: Um estudo multicaso. Contextus: Revista Contemporânea de Economia e Gestão. Fortaleza, v. 9, n. 1, jan.-jun. 2011.

PEREIRA, Flavia Aparecida B; MELLO, Luciana Torres C. de; QUEIROZ, Fernanda Cristina B. P. A Gestão de Conhecimento na Modernização da Gestão Universitária: o caso da UERN (RN). In: XI COLÓQUIO INTERNACIONAL SOBRE GESTÃO UNIVERSITÁRIA NA AMÉRICA DO SUL, 2011, Florianópolis. Anais... Florianópolis: IGLU, 2011.

INEP. Censo da educação superior: 2011 - resumo técnico. Brasília: Instituto Nacional de Estudos e Pesquisas Educacionais Anísio Teixeira, 2013. Disponível em:

$<$ http://download.inep.gov.br/download/superior/censo/2011/resumo_tecnico_censo_educaca o_superior_2011.pdf $>$. Acesso em: 10 jul. 2013.

SANTOS, Leomar dos; BRONNEMANN, Marcia Regina. Desafios da gestão em instituições de ensino superior: um estudo de caso a partir da percepção de diretores de centro de uma IES pública do sul do Brasil. GUAL: Revista Gestão Universitária na América Latina.

Florianópolis, v. 6, n. 1, p. 01-21, jan. 2013.

VENTURINI, Jonas Cardona; PEREIRA, Breno Augusto D.; MORALES, Ronaldo; FLECK, Carolina Freddo; JUNIOR, Zeno Batistella; NAGEL, Mateus de Brito. Percepção da avaliação: um retrato da gestão pública em uma instituição de ensino superior. RAP: Revista de Administração Pública. Rio de Janeiro, 44(1): 31-53, JAN.-FEV. 2010.

VIDOR, Gabriel; FOGLIATTO, Flávio Sanson; CATEN, Carla Ten. Construção de Instrumento para Avaliação de Cursos de Pós-graduação. Avaliação: Revista da Avaliação da Educação Superior. Campinas; Sorocaba, v. 18, n. 1, p. 181-199, mar. 2013. 